Hybrid Micro-Electro-Mechanical Systems for Highly Reliable and Selective Characterization of Tank Waste

(DE-FG07-01ER62718)

Final Technical Progress Report for the Period

September 15, 2001 - September 14, 2005

Prepared for

U.S. Department of Energy

Environmental Management Science Program 


\title{
Hybrid Micro-Electro-Mechanical Systems for Highly Reliable and Selective Characterization of Tank Waste (DE-FG07-01ER62718)
}

\author{
Panos G. Datskos (ORNL), Michael J. Sepaniak (UT); Principal Investigators \\ Nickolay Lavrik, Pampa Dutta, Mustafa Culha; Postdoctoral Associates \\ Chris Tipple, Jeremy Headrick, Joseph Payne, Jim Patton, Kathleeen Giesfeldt; Graduate Students
}

\section{Research Objective:}

The main objective of this research program is to develop robust and reliable micro-electro-mechanical sensing systems, based on microcantilevers (MCs), that can operate in liquid environments with high levels of sensitivity and selectivity. The chemical responses of MCs result from analyte-induced differential stress at the cantilever surfaces. We aim to employ various surface nanostructuring strategies that enhance these stresses and hence the degree of static bending of the cantilevers. Receptor phases as self assembled monolayers (SAMs) and thin films are being synthesized and tested to provide selectivity. Selectivity is chemically enhanced by using different phases on individual MCs in arrays and by adding a spectroscopic component, surface enhanced Raman spectrometry (SERS), in hybrid approaches to sensing. Significant progress was made in tasks that were listed in the work plan for DOE EMSP project "Hybrid Micro-Electro-Mechanical Systems for Highly Reliable and Selective Characterization of Tank Waste." Several project areas are listed below and discussed and referenced to our literature on the topics.

\section{Disordered and Ordered Nanostructuring Technologies for Improved Microcantilever Transduction}

The use of microcantilevers (MCs) as chemical and biological sensors has increased in recent years. The need to make chemical and biological measurement in aqueous environments highlights a limitation of smooth surfaced MCs. The bending of a MC is caused by the creation of a differential surface stress between its two sides as described by the Stoney equation. The modulation of surface stress is related to the initial interfacial energy of the surface by the Shuttleworth equation; ${ }^{1}$ consult Reference 1 for details. In aqueous environments the initial interfacial energy of a smooth surface is small and this limits the sensitivity of the MC. The interfacial energy is further reduced when an organic film is applied to the gold surface. Table 1 shows a comparison of interfacial energies of different interfaces with a Au surface.

The use of chemically-selective thin film coatings has been shown to enhance both the chemical selectivity and sensitivity of MC chemical sensors. As an analyte absorbs into the coating, the coating can swell or contract causing an in-plane stress at the associated microcantilever surface. However, much of the stress upon absorption of an analyte may be lost through slippage of the chemical coatings on the MC surface, or through relaxation of the coating in a manner that minimizes stress to the cantilever. Both ordered and disordered structural modifications of MC chemical sensors can improve the stress transduction between the chemical receptor phase and the microcantilever, and increases the effective surface area, thus improving the sensitivity. We investigate the improvement of sensitivity of MCs using both disordered and ordered structural modifications to the MC surface.

\begin{tabular}{ll}
\hline \hline \multicolumn{2}{l}{ Table 1. Interfacial Energies of Different Interfaces with a Gold Surface } \\
\hline \hline Interface & Interfacial Energy $(\mathbf{m N} / \mathbf{m})$ \\
\hline Au-Air & $>1000$ \\
Au-Water & $\sim 150$ \\
Au-Organic-Water & $\sim 25$ \\
\hline \hline
\end{tabular}

Microcantilever surfaces were modified in three manners. The first method involves the codeposition of gold and silver by physical vapor deposition, followed by a chemical etching step to remove the silver from the surface. $^{1-3}$ This "dealloyed" method produces a highly disordered nanostructured surface on one side of the MC. The nanostucturing process increased the available surface area and creates a quasi-3-D structure that is 
colloidal in nature (Figure 1A). This surface was then coated with different receptor phases as either a selfassembled monolayer (SAM) or a thin film of a vapor deposited phase. The phases were either commercially available calixarenes or newly synthesized cyclodextrin phases prepared as part of this project (see below). The responses of these MCs to 2,7-dihydroxynaphthalene is shown in Figure 1B. The dealloyed MC gives larger responses than smooth surface MCs and thicker films give the largest responses. It is important to note that the response for the $50 \mathrm{~nm}$ dealloyed film is to a 4-fold lower concentration than the response shown for the $18 \mathrm{~nm}$ film on dealloyed and smooth surfaces. In some instances the enahancement in response from nanostructuring exceeded 100-fold. ${ }^{3}$
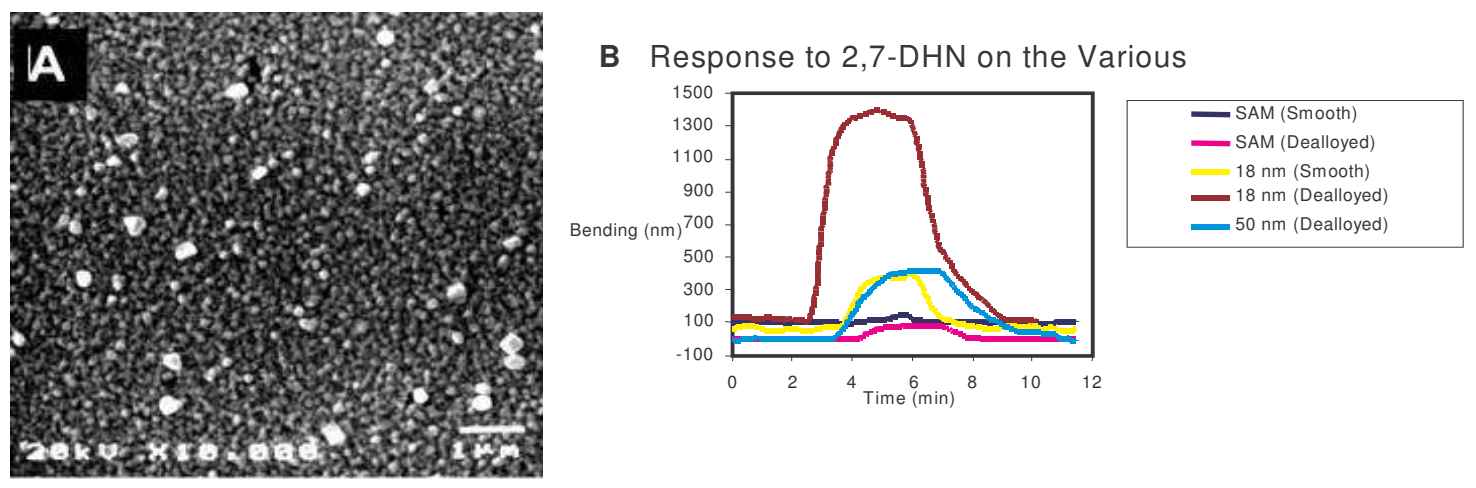

Figure 1. A) SEM image of dealloyed surface. B) Responses to 2,7-DHN

While the dealloying process produces a highly disordered nanostructured surface, the second manner in which MC surfaces were modified involved creating an ordered nanostructured surface by milling grooves across the width of the MC using a focused ion beam (FIB) mill. ${ }^{4}$ An example of a milled lever is shown in Figure 2A. As seen in the figure the grooves are positioned approximately every $800 \mathrm{~nm}$ and are roughly 400 $\mathrm{nm}$ wide. Two different groove depths were milled, 200 and 400nm deep. The grooves covered the $100 \mu \mathrm{m}$ nearest the base of the $200 \mu \mathrm{m}$ long MCs. The entire MC was not milled in order to leave an area to efficiently reflect a readout laser beam. The MCs were coated with a thin film of cyclodextrin phase by physical vapor deposition and responses to 2,3-dihydroxynaphthalene measured. As seen in Figure 2B the grooved MC outperformed the smooth one. The improvement is a combined effect of confining the receptor phase in the grooves and a reduced spring constant.

The final method of preparing surface modified MCs produces ordered grooves similar to those produced by method 2. The method involves doping regions of the silicon MCs with gallium ion using the FIB. The MCs are then etched in a $60 \% \mathrm{w} / \mathrm{w} \mathrm{KOH}$ solution. The doped regions of silicon etch at a slower rate than the undoped regions producing V-shaped grooves. In addition to the unique shape of the regular surface structures the processing time is greatly diminished relative to the straight milling procedure. These structures are currently under investigation.
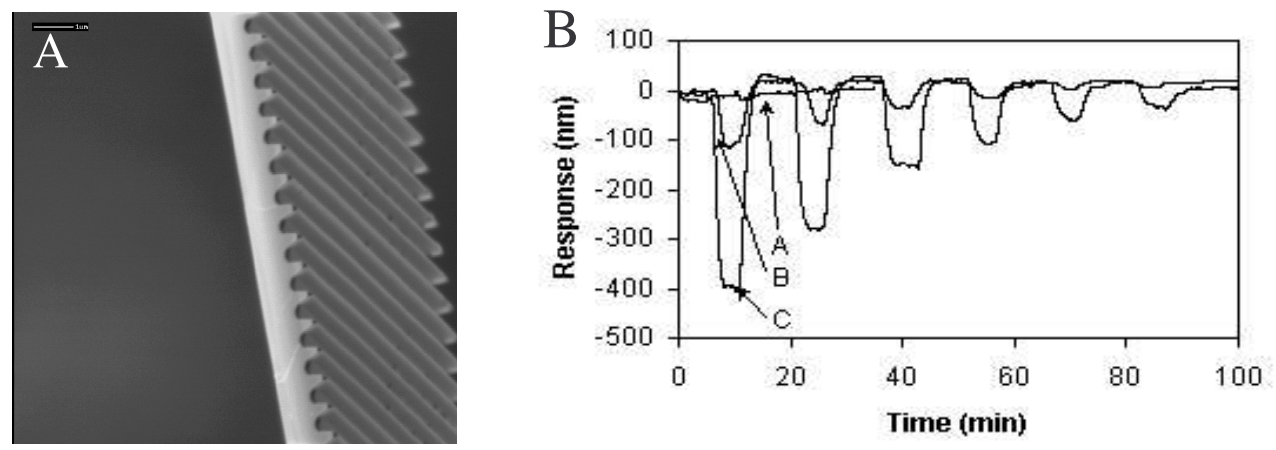

Figure 2. A) Grooved Microcantilever with 400nm deep grooves. B) Responses for surface modified microcantilevers to decreasing concentrations (left to right) of 2,3-DHN. (A) Ungrooved, (B) $200 \mathrm{~nm}$ deep, (C) 400nm deep grooves. 


\section{Exploring Microcantilever Compatible Surface Enhanced Raman Scattering (SERS) Substrates for Hybrid Sensors}

Up to this point little work has been done with MCs to allow measurements in complex mixtures or to use MCs to acquire structural information. One technique that could be used to obtain structural information is Raman. Both MC measurements and Raman spectroscopy techniques use lasers to probe microscopic regions of a surface. In the case of MCs the laser is used to monitor the bending, while in Raman it is used to cause an excited state of the molecules absorbed to the surface. While Raman spectra offer a wealth of qualitative information the conventional Raman technique suffers from poor sensitivity. Fortunately, Raman sensitivity can be greatly enhanced by resonance and surface effects. In the latter case, it is found that chemical and electromagnetic enhancement effects produce large increases in Raman signals when analyte are adsorbed onto or very close to certain roughened metal surfaces. ${ }^{5}$ Since roughened surfaces such as the dealloyed MCs discussed above provide for enhanced bending response, a goal of this work was to determine if these surfaces were also SERS active. The creation of a hybrid MC-SERS sensor would have specificity of receptor binding and be able to acquire vibrational signatures of the bound analytes on the surface of the MC.

To produce a hybrid MC-SERS sensor a good SERS substrate needs to be found that can be used with MCs. We have explored several methods of preparing SERS active substrates on MC chips. The first SERS substrate investigated was the nanostructured, dealloyed film used previously to enhance MC bending. The dealloyed films gave responses that were as good as traditional silver island films on glass when used as SERS substrates. Figure 3 shows several spectra of thiolated compounds on a dealloyed gold surface. ${ }^{6,7}$ Excitation was via the $633 \mathrm{~nm}$ output of a He-Ne laser. The same dealloyed surface showed significant improvement in SERS activity when a thin layer of silver (which better matches the laser wavelength) was coated over the dealloyed surface. Another substrate used was a polydimethylsiloxane (PDMS) overcoated with a silver island film. This substrate shows even greater enhancement over the other types of substrates investigated.

To date all of these substrates have shown drawbacks that include photodegradation of the SERS active substrate upon long exposure times and long desorption times of adsorbed molecules from the substrate. We discovered that the photodegredation problem can be overcome by very effectively rastering the laser spot onto new areas of the SERS substrate to prevent photodegredation without loss of signal. ${ }^{8}$ The long desorption times of adsorbed molecules is a more complex problem that may require the development of thermal desorption methods.

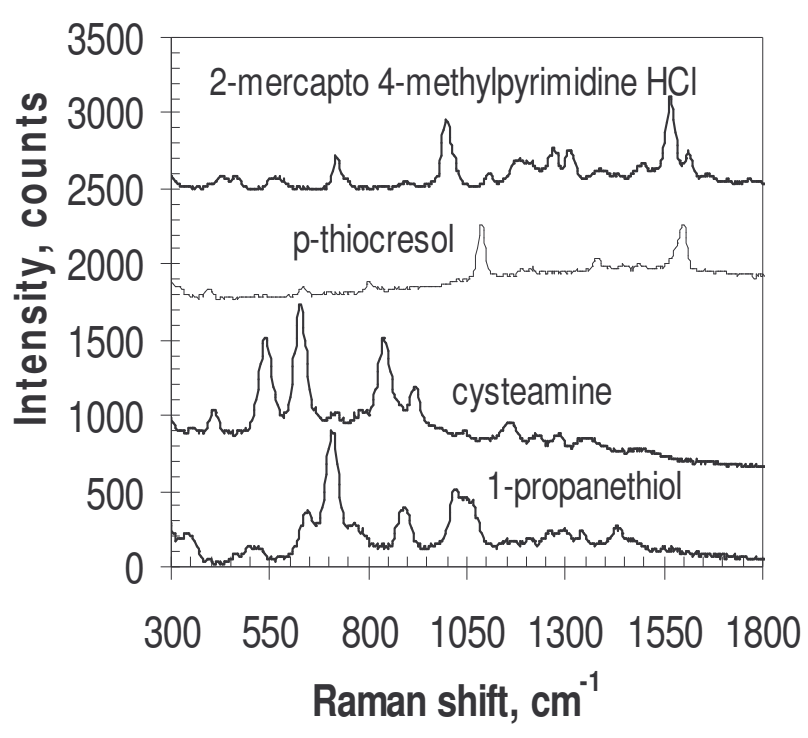

\section{Differential Microcantilever Readout for Improved Reliability and Robustness in Sensing Applications}

The current trend in analytical sensing systems is to increase sensitivity and selectivity by employing array based devices. MCs are easily miniaturized and can be readily integrated into sensor arrays. These arrays achieve greater sensitivity and a higher degree of selectivity than using a single cantilever due to the reduction of background noise and the use of multiple chemically selective coatings, respectively. There are several key sources of noise that have plagued MC based sensing systems since their infancy. Temperature drifts, changes in refractive index, flow rate changes, and mechanical sources of noise are but a few. While some strides have been made in the area of expanding the number of available selective coatings that can be employed using MC 
sensing systems, there are very few groups employing array based MC sensing systems. A goal of our work was to develop a simple, synchronous dual laser differential optical bending readout mode for MC sensing.

The differential based system employs two lasers that are modulated between two adjacent MCs (see Figure 4). The reflected laser beams are then directed onto a position sensitive detector and MC bending is measured in an AC mode of operation using a lock in detector. This system was developed and compared to simple static bending measurements. Various factors that affect the performance of MC based chemical sensors have been studied using both systems. These effects were studied using non-chemically coated, nanostructured silicon array MCs so that there would be no chemical difference between the MCs that might lead to differences in response. The effect of these factors (i.e. temperature drift, refractive index, flow rate, ionic strength, mechanical noise, etc) has been minimized by using the differential based system. In most cases, the response caused by these factors in the simple static bending system has been decreased by a factor of 10 when using the differential system. Figures 5 shows the effects of sample flow rate on baseline stability with the differential and simple static bending modes of readout. ${ }^{9}$

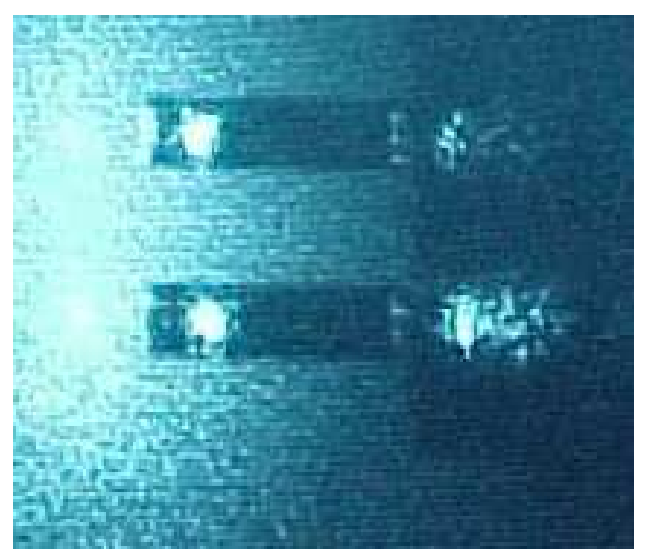

Figure 4. Rectangular MCs used in our studies.

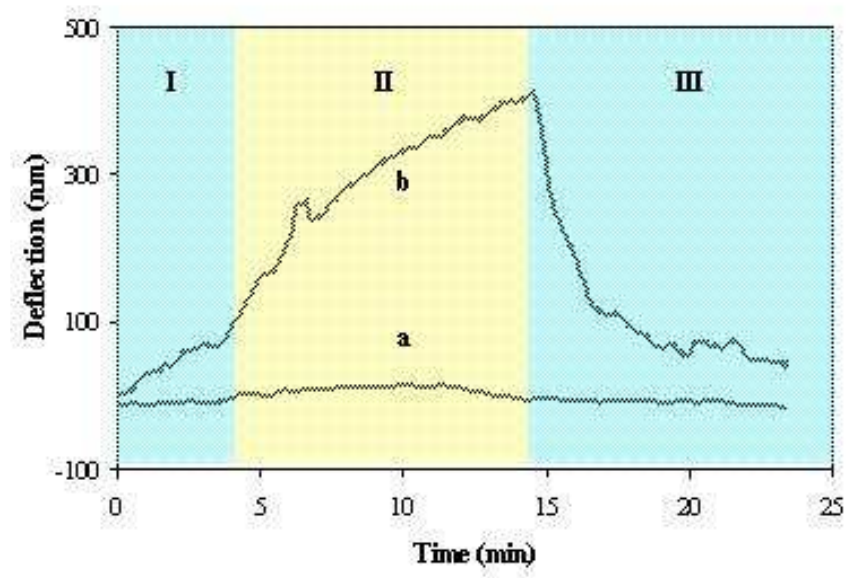

Figure 5. Illustration of the improvement in baseline stability when a simple single cantilever optical arrangement (DC output), a, is replaced by a differential (AC output) arrangement, b. Baselines were recorded under stagnant (region II) and flow at $1.0 \mathrm{~mL} / \mathrm{minute}$ (regions I and III) conditions.

\section{Methods of Differentially Applying Novel Macrocycle Receptors to MC Surfaces for Selective Measurements of Mixtures}

As stated above, MCs are well suited to be used as elements in sensor arrays. These arrays lead to a higher degree of selectivity than can be achieved using a single cantilever due to the use of multiple chemically selective layers. In order to impart selectivity when MCs are used in analytical sensing, these chemically selective receptor phases are immobilized on one of the sides of the cantilever. Limiting the phase to one side of the MC is important because differential modulation of the surface stress is achieved by using asymmetric MCs wherein one side of the cantilever preferentially interacts with the analyte via surface adsorption or absorption into a thin chemical film on the MC surface. Ideally, this interaction of the analyte with the receptor phase is reversible and exhibits reasonable kinetics for sensing applications. While it is reasonably simple to coat all of the MCs in an array with the same receptor phase, it is quite challenging to coat an individual MC in an array with a receptor phase. Therefore, progress in developing simple and reliable methods of coating each MC in an array with a different receptor phase is vital to the application of these array-based sensors. Goals of this work include expanding the array of selective phases that are compatible with MCs and developing methods to differentially coat those phases onto MC array. 
Since cyclodextrins have been shown to exhibit very good selectivity based on size, shape, and chemical properties we have development methods to functionalize them for use with MCs. The cyclodextrins are tea cup-shaped macrocycle sugars with two secondary hydroxyl groups on each sugar at the upper rim and one primary hydroxyl groups on each sugar at the bottom. We have functionalize the bottom with thio groups to bond to gold coated cantilevers. ${ }^{1}$ In addition, the scheme shown in Figure 6 was used to functionalize both the primary and secondary hydroxyls to yield a structure that is thermally labile and stable. ${ }^{10}$ The former are used as SAMs and the latter as thin films.
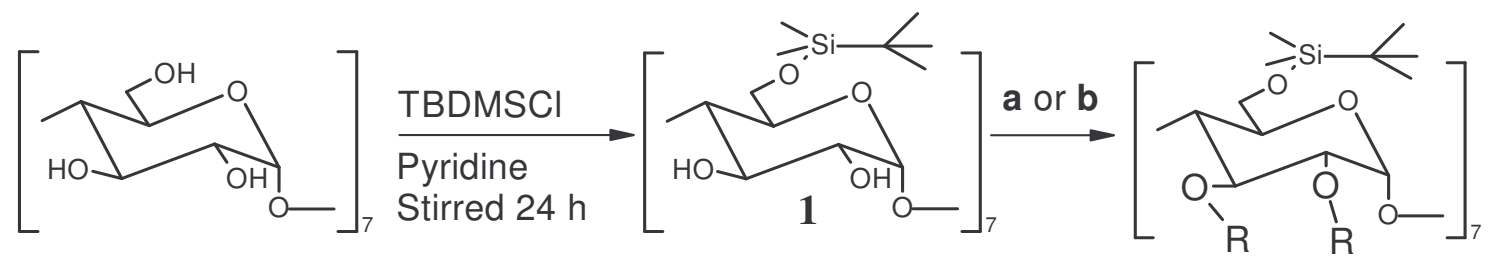

a) $\mathrm{NaH}, \mathrm{RI}, \mathrm{THF}, 24 \mathrm{~h}$; b)Acetic anhydride, Pyridine, Reflux $4 \mathrm{~h} 100^{\circ} \mathrm{C}$

a) $\mathrm{R}=\mathrm{CH}_{3}-$

b) $\mathrm{R}=\mathrm{CH}_{3} \mathrm{COO}$ -

Figure 6. Synthetic scheme to create a cyclodextrin phase that can be vapor deposited onto MCs

We report herein two approaches aimed at selectively coating single MCs in an array. MCs have been coated with various calix[n]arene and functionalized cyclodextrin films using the vapor deposition through a mask procedure. Figure 7 shows the alignment of a MC over the slit used in this procedure. As can be seen in the figure, a single MC is clearly seen through the mask, while neither of the adjacent MCs is visible. This ensures that neither of the two adjacent MCs have been coated with the phase that has been applied to the MC shown. This coating approach has been applied to the measurement of a mixture of quinoxaline and 8hydroxyquinoline in solution by coating two adjacent MCs with calix[6]arene and calix[8]arene, respectively.

Calibration curves were obtained for each analyte independently using each phase and response factors were calculated using this data. An unknown mixture of the two analytes was then prepared and measured using the two calix[n]arene phases from above. Figure 8 shows calibration plots for the analysis of quinoxaline (quin) and 8-hydroxyquinoline (hq) on the two different types of MCs used, respectively. By using the data presented in Figure 8, the individual components in an unknown mixture could be quantified with associated errors of only $20.7 \%$ and $1.6 \%$ for the quantification of 8 -hydroxyquinoline and quinoxaline, respectively.

MCs have also been coated with various phases utilizing liquid phase reactions with the surface. This approach utilizes a microcapillary filled with the phase to be reacted with the surface. The technique has been used to coat MCs with thiolated compounds and compounds of biological interest as well.

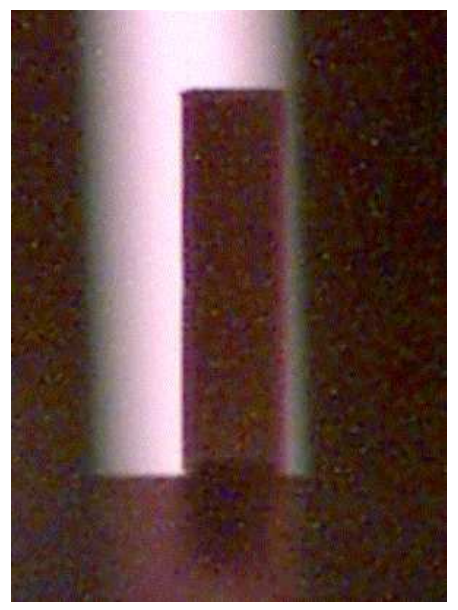

Figure 7. $100 \mu \mathrm{m}$ wide MC from array seen through vapor deposition mask.

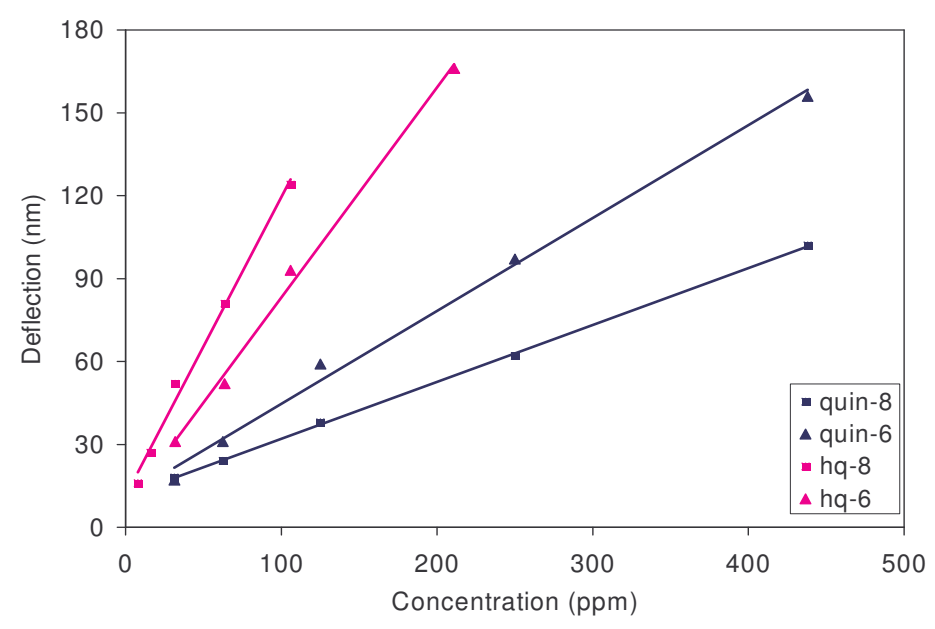

Figure 8. Calibration data for quinoxaline and 8-hydroxyquinoline on two calix[n]arene phases. 


\section{Highly-Selective Sensing Based on MC Antibody-Mediated Nanomechanics}

As an impressive example of biologically attained selectivity, we recently demonstrated that chiral discrimination of amino acid enantiomers is possible using anti-D-amino acid and anti-L-amino acid antibodies immobilized on the surfaces of nanostructured MC. ${ }^{11}$ It was possible to measure the specific enantiomer in the presence of large excesses of the nonspecific enantiomer (Fig. 9). While the robustness of bioaffinity systems may be questioned in the context of tank waste measurements, very high levels of biologically-inspired selectivity should be possible using molecularly imprinted polymer phases.

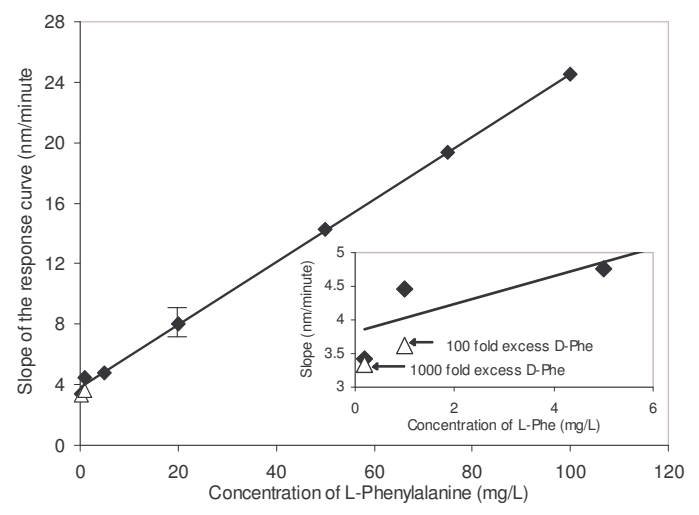

Figure 9. Analytical data that includes a calibration curve $\left(r^{2}=0.999\right)$ based on the slope of the response curve obtained with an anti-Lamino acid functionalized MC. The cantilever was exposed to different concentrations of Lphenylalanine as well as to mixtures containing a 100 - and 1,000-fold excess of Dphenylalanine.

\section{Response Signatures from Differentially Functionalized MC Arrays}

In recent studies, we have developed MCs, nanostructured on one side and configured in spatially-dense arrays, for the sensitive detection of organic vapor mixtures. ${ }^{\mathbf{1 2}}$ MCs singularly coated with seven different molecular recognition phases (MRPs) exhibit differential selectivity and response. The phases are films of nanodimensional thickness which are deposited by physical vapor deposition (PVD) method and have been structurally characterized post deposition. The phases include polymers, GC phases, and macrocyclic receptors (see reference 12) Analyte-induced bending of the MCs in arrays is monitored using beam displacements of vertical cavity surface emitting lasers using a time multiplexing scheme. ${ }^{12}$ The magnitudes and shapes of the array response profiles are unique to the analytes tested (Figure 10). Also, larger responses to the analytes are realized with increasing layer thickness.
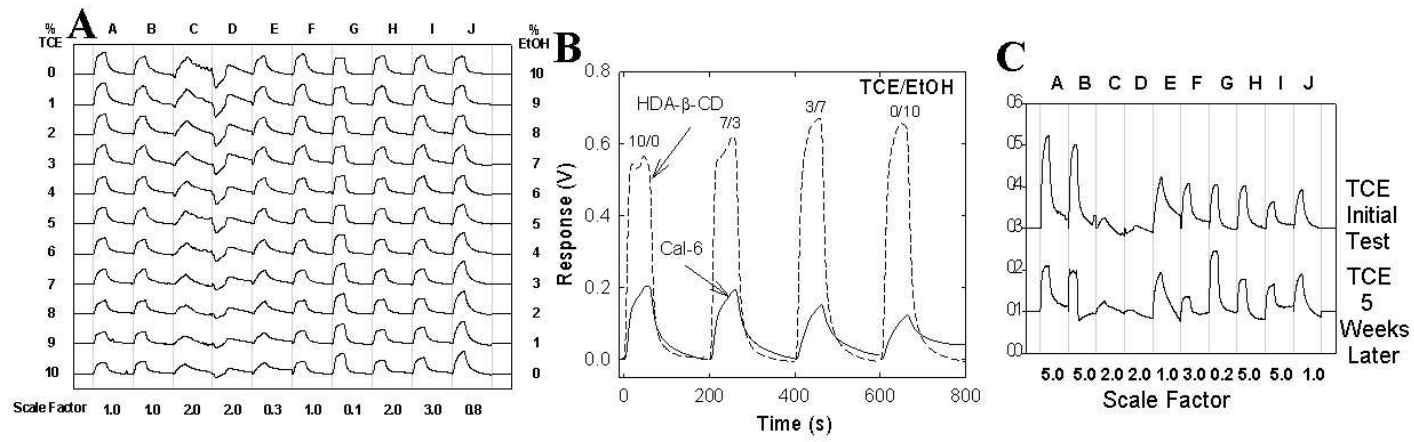

Figure 10. (A) - Responses of the MC array to $10 \%$ (v/v) of TCE and ethanol mixture diluted with nitrogen where TCE and ethanol concentrations were varied within $0-10 \%$ of room te mperature head space concentration in the mixture. Each grid corresponds to 200 second response of each phase among 10 different MRPs from A to J. Where A - propanethiol functionalized dealloyed; B - CD (350nm); C-PDPP(452n m); D-PIB(450n m); E - Cal-6(400n m); $\mathrm{F}$ - same as A; G - same as B(450n m); H - Squalane (450n m); I - PECH(450n m); J - Cal-4(400n m). Response scaling factors are shown near bottom of the figure. (B) - The uniqueness of the analyte signatures are highlighted for a two MRP subset from A. (C) - Comparis on of the response of the MC array to $10 \%$ (v/v) TCE with the response of an identical measurement obtained 5 weeks later after multip le measurements. Each grid corresponds to 200 second response of each phase among 10 different MRPs from A to $\mathrm{J}$, where A to $\mathrm{J}$ is same as in $\mathbf{A}$. 


\section{Literature Cited and Information Access:}

1. N.V. Lavrik, C. Tipple, P. Datkos, and M. Sepaniak, "Enhanced Chemi-Mechanical Transduction at Nanostructured Interfaces," Chem. Phys. Lett., 336, 371 (2001).

2. N.V. Lavrik, C.A. Tipple, P.G. Datskos, and M.J. Sepaniak, "Gold Nano-Structures for Transduction of Biomolecular Interactions into Micrometer Scale Movement," Biomedical Microdevices, 3, 35-44 (2001).

3. C.A. Tipple, N.V. Lavrik, M. Culha, J. Headrick, P.G. Datskos, and M.J. Sepaniak, "Nanostuctured Microcantilevers With Functionalized Cyclodextrin Receptor Phases: Self Assembled Monolayers and Vapor Deposited Films," Anal. Chem. 74, 3118-3126 (2002).

4. J. J. Headrick, M.J. Sepaniak, N.V. Lavrik, and P.G. Datskos, "Enhancing Chemi-Mechanical Transduction in Microcantiliver Chemical Sensing by Surface Modification," Ultramicroscopy, submitted.

5. R. J. Hinde, M.J. Sepaniak. R.N. Compton, N.V. Lavrik, and J. Nordling, "Surface Enhanced Raman Scattering Under Liquid Nitrogen," Chem. Phys. Lett., 339, 167 -173 (2001).

6. M. J. Sepaniak, N.V. Lavrik, C.A. Tipple, and P. G. Datskos, "Hybrid Nanostructured Microcantilevers for Enhanced Chemimechanical Transduction and SERS", Micro Total Analysis Systems, J.M Ramsey and A. van den Berg, Eds, Kluwer Academic Publishers, London, 450-452 (2001).

7. N. V. Lavrik, C.A. Tipple, P.G. Datskos, and M.J. Sepaniak, "Sensing and Actuating Functionality of Hybrid MEMS Combining Enhanced Chemi-Mechanical Transduction with SERS," Proceedings of SPIE-Soc. Opt. Eng., 4560, 152-161 (2001).

8. M. A. De Jesus, K. S. Giesfeldt, and M.J. Sepaniak, "The Development of a Substrate Translation Techniques to minimize the Adverse Effect of Laser Irradiation Effects on Surface Enhanced Raman Scattering Spectra,” Appl. Spectrosc..57, 428-438 (2003).

9. M. J. Sepaniak, P.G. Datskos, and N.V. Lavrik, "Microcantilever Transducers: a New Approach in Sensor Technology” Anal. Chem. 74, 568A (2002).

10. M. Culha, N.V. Lavrik, F.M. Schell, C.A. Tipple, and M.J. Sepaniak, "Characterization of Volatile, Hydrophobic Cyclodextrin Derivatives as Thin Films for Sensor Applications," Sensors and Actuators $B$, 92, 171-180 (2003).

11. P. Dutta, C.A. Tipple, N.V. Lavrik, P.G. Datskos, O. Hofstetter, and M.J. Sepaniak, "Enantioselective Sensors Based On Antibody-Mediated Nanomechanics," Anal.Chem., 75, 2342-2348 (2003).

12. P. Dutta, L.R. Senesac, N.V. Lavrik, P.G. Datskos, and M.J. Sepaniak, "Response Signatures for nanostructured, Optically Probed, Functionalized Microcantilever Sensing Arrays," Sensors Lett. 2, 1-8, (2004). 